Original Research Article

\title{
A retrospective analysis of adverse drug reaction reported in a tertiary care hospital
}

\author{
Kirti Saxena*, Chaitali Tailor, Chaitali Mehta, Paresh Gajera, S. K. Srivastava
}

Department of Pharmacology, Surat Municipal Institute of Medical Education and Research, Surat, Gujarat, India

Received: 20 February 2017 Accepted: 28 March 2017

*Correspondence to:

Dr. Kirti Saxena,

Email: saxenadrp@gmail.com

Copyright: (C) the author(s), publisher and licensee Medip Academy. This is an openaccess article distributed under the terms of the Creative Commons Attribution NonCommercial License, which permits unrestricted noncommercial use, distribution, and reproduction in any medium, provided the original work is properly cited.

\begin{abstract}
Background: The adverse drug reactions (ADRs) reported to pharmacovigilance centre in tertiary care hospital was analysed to find out the incidence and causality.

Methods: This was a retrospective study to analyse the ADR reported at pharmacovigilance centre after ethical clearance from Institutional Ethic Committee (IEC). ADR data were analysed and ADRs were categorized as department-wise, system affected and causative drug. The causality of each ADR was assessed by WHO-UMC scale.

Results: The majority of patients who had suffered from ADRs were between 19-64 years of age (94.2\%) and male patients $(58.6 \%)$ were affected more than female $(41.4 \%)$. Pulmonary medicine department has reported highest number of ADR followed by dermatology department. Skin (46.5\%) was most affected system followed by gastrointestinal (30.45\%), CNS $(21.26 \%)$, respiratory $(9.0 \%)$ and remaining systems. Rifampicin $(13.79 \%)$ shows the largest numbers of ADR followed by zidovudine (13.21\%), nevirapine (12.64\%) and diclofenac sodium $(8.0 \%)$. The maximum ADRs reported were probable $(94.8 \%)$ followed by possible $(5.2 \%)$.

Conclusions: In conclusion, the skin was most affected system followed by gastrointestinal, central nervous and respiratory system. Rifampicin has caused maximum ADRs followed by zidovudine, nevirapine and diclofenac sodium. The causality analyses showed that majority of ADRs were probable (94.8\%) while remaining falls in possible $(5.2 \%)$ category.
\end{abstract}

Keywords: Adverse drug reaction, Pharmacovigilance, UMC scale

\section{INTRODUCTION}

Adverse drug reaction (ADR) as defined by WHO are "any noxious, unintended, undesired effect that occurs at dosage used in human for prophylaxis, diagnosis and therapy". ${ }^{1}$ There is need to strengthen the ADR reporting system in India because of underreporting of ADR $(<1 \%)$ as compared to other countries. ${ }^{2}$ This may be due to multiple factor including lack of awareness among health care professionals and poor post marketing surveillance by pharmaceuticals.

ADR reporting is an important component of monitoring and evaluation activity in hospital. ${ }^{3,4}$ In November 2004 CDSCO, ministry of health and family welfare government of India launched the National Pharmacovigilance programme (NPP) which was converted to Pharmacovigilance Programme of India
(PvPI) in 2010 to monitor the ADRs that include government or private sector. ${ }^{5}$ The PvPI aims at safe use of medicine. Thus, it is important to have ADR data of all the drugs, so that necessary measures can be taken in this regard. The present study was planned to analyse ADRs reported in tertiary care hospital.

The objective of the study was to retrospectively analyse ADRs reported to Pharmacovigilance centre in a tertiary care hospital. Analysis was done to find out the incidence and causality of reported ADR.

\section{METHODS}

This was a retrospective study. The analysis of ADR reported at Pharmacovigilance centre in tertiary care hospital during last one and half year (from 1-1-2014 to 30-4-2015) was done. The clearance from institutional 
ethical committee was taken before the commencement of study. Confidentiality was maintained at all the level.

Completed ADR reporting forms submitted to ADR monitoring centre were analysed. The details filled up in the form were checked for mandatory parameters including patient's detail, type of ADR, drugs causing ADR, etc.

ADRs were analysed and results were categorized as follows:

- Demographic details

- Department-wise

- System affected

- Causative drug

- Causality of ADRs

WHO-UMC scale adopted by PvPI was used for causality assessment.

Results were presented in tabular form, Pie chart and bar diagram.

\section{RESULTS}

A total of 174 ADRs were detected, documented and reported during the last one and half year.

The majority of patients who had suffered from ADRs were between 19-64 yrs. of age (94.2\%) of total ADRs followed by $0-18$ yrs. $(5.2 \%)$. There was only $0.6 \%$ of reporting in the age group more than 65 yrs. The studies also revealed male patient $(58.6 \%)$ were affected more than female $(41.4 \%)$ (Table 1$)$.

Table 1: Demographic details.

\begin{tabular}{|lll|}
\hline Gender & Total no. 174 & Percentage \\
\hline Male & 102 & 58.6 \\
\hline Female & 52 & 41.4 \\
\hline Age distribution & & \\
\hline 0-18 yrs & 9 & 5.2 \\
\hline 19-64 yrs & 164 & 94.2 \\
\hline 65 yrs and above & 1 & 0.6 \\
\hline
\end{tabular}

The analysis showed that Pulmonary medicine department reported highest number of ADRs (31.6\%) followed by dermatology department $(24.1 \%)$. The least number of reporting were from surgery department $(0.6 \%)$ (Table 2).

On analysis of system affected the skin was most commonly affected $(46.5 \%)$ followed by gastrointestinal (GI) tract (30.45\%), CNS $(21.26 \%)$ and respiratory system $(9.19 \%)$. The involvement of genitourinary and haemopoetic systems was $(1.72 \%$ each). The endocrine system (thyroid) was affected in $(0.57 \%)$ of patients (Table 3).

Table 2: Department wise ADR report.

\begin{tabular}{|lll|}
\hline Departments & No. of ADR $(\mathbf{n = 1 7 4})$ & Percentage \\
\hline TB and Chest & 55 & 31.6 \\
\hline Dermatology & 42 & 24.1 \\
\hline ART center & 36 & 20.7 \\
\hline Psychiatric & 17 & 9.8 \\
\hline Medicine & 11 & 6.3 \\
\hline Obs. and Gynec. & 5 & 2.9 \\
\hline Pediatric & 5 & 2.9 \\
\hline Orthopedic & 2 & 1.1 \\
\hline Surgery & 1 & 0.6 \\
\hline
\end{tabular}

Table 3: System wise ADR reports.

\begin{tabular}{|lll|} 
Systems & $\begin{array}{l}\text { No. of Patient } \\
\text { (Total=174) }\end{array}$ & $\begin{array}{l}\% \text { of } \\
\text { patients }\end{array}$ \\
\hline Skin & 81 & 46.55 \\
\hline GIT & 53 & 30.45 \\
\hline CNS & 37 & 21.26 \\
\hline Respiratory System & 16 & 9.19 \\
\hline Genitourinary & 3 & 1.72 \\
\hline Anemia & 3 & 1.72 \\
\hline Endocrine & 1 & 0.57 \\
\hline
\end{tabular}

The details of ADR related to each system are mentioned in Table 4.

In our study the rifampicin shows the largest number of ADRs (13.79\%) of total followed by zidovudine (13.21\%) then nevirapine (12.64\%). Diclofenac sodium also shows significant no. of ADR $(8.0 \%)$ (Table 5).

The maximum ADRs reported were probable (94.8\%). This was followed by possible (5.2\%). No ADR was reported from any other category of WHO-UMC scale (Table 6). ${ }^{5}$

\section{DISCUSSION}

In this study, the Patient suffering from ADRs were between 19-64 years of age in which males $(58.6 \%)$ were more affected than females $(41.4 \%)$. In contrast to A. P Gor's study which showed that the sex of the patients did not affect the incidence rate of ADR, our study showed a higher prevalence among males. ${ }^{5}$

Patidar et al observed that the occurrence of ADRs was $(45.94 \%)$ in men and $(54.05 \%)$ in women. ${ }^{6}$ Other studies by Sriram et al, Richa, VR Tandon et al reported that males have greater risk of ADR than females. ${ }^{12,15}$ There are various factors like age of patients, gender, number of drug taken, duration of hospital admission, genetic factors, ethnicity, dietary, and environmental factors affecting the ADR incidence. 
Table 4: Clinical feature wise ADR reports.

\begin{tabular}{|c|c|c|c|}
\hline System & ADR & Drug & $\begin{array}{l}\text { No. of } \\
\text { patient }\end{array}$ \\
\hline \multirow{7}{*}{ Skin } & Multiple Scaly Plaque like lesion & Losartan, Atenolol & 2 \\
\hline & Angioedema & $\begin{array}{l}\text { Iron Sucrose, Diclofenac Sodium, Ibuprofen, } \\
\text { Ceftriaxone, Artemether, Amoxicillin + } \\
\text { Clavulanic acid }\end{array}$ & 11 \\
\hline & Hyperpigmented patches & Cotrimoxazole & 1 \\
\hline & $\begin{array}{l}\text { Multiple Erythromatous Maculopapular } \\
\text { Rash }\end{array}$ & $\begin{array}{l}\text { Nevirapine, Cotrimoxazole, Cefoperazone, } \\
\text { Azithromycin, Ibuprofen, Phenytoin Sodium, } \\
\text { Multivitamin Tablets Isoniazid, Isoniazid+ } \\
\text { Rifampicin, Diclofenac Sodium + Paracetamol, } \\
\text { Zidovudine }\end{array}$ & 43 \\
\hline & Exfoliative Dermatitis & Zidovudine+ Lamivudine+Nevirapine & 10 \\
\hline & Urticaria (itching) & $\begin{array}{l}\text { Dilofenac Sodium, Amoxicillin, Navirapine, } \\
\text { Abacavir, Topiramate }\end{array}$ & 13 \\
\hline & $\begin{array}{l}\text { Steven Johnson Syndrome (Cutaneous } \\
\text { ulceration Epidermal Necrosis) }\end{array}$ & Lamotrigine & 1 \\
\hline \multirow[t]{2}{*}{ GIT } & $\begin{array}{l}\text { Gastritis ((Gastric Intolerance, Vomiting } \\
\text { Nausea, Abdominal Pain and Decrease } \\
\text { appetite) }\end{array}$ & $\begin{array}{l}\text { Zidovudine, Nevirapine, Lorazepam, } \\
\text { Capreomycin, Levofloxacin, Pyrizinamide }\end{array}$ & 52 \\
\hline & Jaundice & AKT (Cat. I) & 1 \\
\hline \multirow{11}{*}{ CNS } & Excessive Sedation (Drowsiness) & Mirtazepine, Olanzepine & 6 \\
\hline & Chills and Rigor & Chloramphenicol & 2 \\
\hline & $\begin{array}{l}\text { Peripheral Neuropathy (Tingling } \\
\text { Numbness) }\end{array}$ & $\begin{array}{l}\text { Stavudine, Stavudine +Nevirapine + } \\
\text { Lamivudine, } \\
\text { Stavudine +Nevirapine + Lamivudine + } \\
\text { Zidovudine }\end{array}$ & 5 \\
\hline & Dizziness & Pregabaline, Phenytoin Sodium, Mirtazepine & 4 \\
\hline & Restlessness (Anxiety) & Pregabaline, Desvenlafaxine, Quetiapine & 4 \\
\hline & Headache & Desvenlafaxine, Etiozolam, Azithromycin & 5 \\
\hline & Tonic Posturing & Amoxicillin + Clavulanic acid & 1 \\
\hline & Insomnia induced psychosis & Isoniazid & 1 \\
\hline & Decrease Hearing & Capreomycin & 1 \\
\hline & Tonic Convulsion & Tetanus & 1 \\
\hline & Fever & $\begin{array}{l}\text { Rifampicin, AKT, Abacavir, Nevirapine, } \\
\text { Isoniazid }\end{array}$ & 7 \\
\hline \multirow[b]{2}{*}{ Respiratory } & Dry Cough (Throat pain, Hemoptosis) & Rifampicin, Isoniazid, Nimesulide & 4 \\
\hline & Angioedema & $\begin{array}{l}\text { Iron Sucrose, Diclofenac sodium, Ibuprofen, } \\
\text { Ceftriaxone, Artemether, Amoxicillin + } \\
\text { Clavulanic acid }\end{array}$ & 11 \\
\hline Endocrine & Hypothyroidism & AKT (Cat. IV) & 1 \\
\hline Genitourinary & Candidiasis. Paracystitis, cystitis & Rifampicin, Tenovir Pyrizinamide & 3 \\
\hline Misc & Anaemia & Ziduvadine & 3 \\
\hline
\end{tabular}

The present study revealed a predominance of adult over paediatric populations. Most of the patients $(94.1 \%)$ were between 19-64 years of age group. The reason for the high morbidity in adult population may be because of multi drug therapy or other disease like hypertension, diabetes, asthma or other chronic diseases. Our finding similar with the finding of Patidar et al, Murphy et al. ${ }^{6,8}$ The most common category associated with ADRs was skin $(46.55 \%)$. This finding is consistent with the study by Coelho et al, Fredy et al, Rajesh et al, but it differs from reports of Suh et al where gastrointestinal manifestations had the highest rate, which was second highest in our study (30.45\%). ${ }^{9-11,13}$ Sriram et al, Rajesh et al Murphy et al, Gor AP, Desai SV, Brennan TA, Leape LL et al, Fredy et al, Leone et al, reported that other antibiotics are the most common classes causing ADRs but in our study patients on anti-tubercular drugs had maximum ADRs. ${ }^{5,712,13}$ The most common drug causing ADR is rifampicin $(13.79 \%)$. It is because of anti-tubercular drug used for long duration (minimum 6 months) as compare to other antibiotics, followed by zidovudine $(13.21 \%)$ and nevirapine (12.64\%). 
Table 5: Drug wise ADR.

\begin{tabular}{|c|c|c|}
\hline Name of Drug & $\begin{array}{l}\text { Frequency of } \\
\text { ADR }\end{array}$ & ADR reported \\
\hline \multicolumn{3}{|l|}{ Antitubercular drugs } \\
\hline AKT & 10 & Gastritis, vomiting nausea anorexia \\
\hline Rifampicin + Isoniazid & 3 & Gastritis, vomiting, abdominal pain \\
\hline Rifampicin & 24 & Gastritis, vomiting, abdominal pain dry cough and fever \\
\hline Isoniazid & 9 & $\begin{array}{l}\text { Maculopapular rashes with fever, chills, psychosis insomnia } \\
\text { weakness }\end{array}$ \\
\hline Pyrizinamide & 4 & Cystitis, weight loss, dyspnoea, anorexia \\
\hline \multicolumn{3}{|l|}{ Antiviral drugs } \\
\hline Nevirapine & 22 & $\begin{array}{l}\text { Multiple erythromatous patch, maculopapular rashes with fever, } \\
\text { erythromatus maculopapular lesions, nausea vomiting headache. }\end{array}$ \\
\hline Zidovudine & 23 & $\begin{array}{l}\text { Exfoliative dermatitis, anaemia, xerosis, fixed drug eruptions, acute } \\
\text { gastritis, severe vomiting abdominal pain }\end{array}$ \\
\hline Acyclovir & 1 & Skin rash and fever \\
\hline Abacavir & 1 & Fever and itching \\
\hline Tenofovir & 1 & Paracystitis \\
\hline Stavudine+Lamivudine & 4 & Tingling Numbness and Peripheral Neuritis \\
\hline Stavudine+Lamivudine+Nevirapine & 1 & Tingling Numbness and Peripheral Neuritis \\
\hline \multicolumn{3}{|l|}{ Antibacterial Agents } \\
\hline Co-trimoxazole & 3 & Hyperpigmented patches, erythromatous Patch \\
\hline Chloramphenicol & 2 & Chills and rigor \\
\hline Azithromycin & 8 & $\begin{array}{l}\text { Gastritis, Headache, abdominal pain, Erythromatous maculopapular } \\
\text { rashes }\end{array}$ \\
\hline Ceftriaxone & 1 & Angioedema \\
\hline Capreomycin & 2 & Decrese hearing, vomiting \\
\hline Levofloxacin & 1 & Chest pain, Decrease appetite \\
\hline Amoxicillin + Clavulanic acid & 4 & Skin lesion, Angioedema and Tonic posture \\
\hline \multirow{2}{*}{\multicolumn{3}{|c|}{ Antimalarial drugs }} \\
\hline & & \\
\hline Artemether & 1 & Angioedema \\
\hline \multicolumn{3}{|l|}{ Antihypertensive drugs } \\
\hline Losartan & 1 & Multiple Scaly Plaque like lesion \\
\hline Atenolol & 1 & Multiple Scaly Plaque like lesion \\
\hline \multicolumn{3}{|l|}{ Analgesic drugs } \\
\hline Ibuprofen & 2 & Maculopapular rashes, angioedema and urticaria \\
\hline Diclofenac Sodium & 13 & $\begin{array}{l}\text { Urticaria, angioedema - itching, tingling, numbness, maculopapular } \\
\text { rashes and tightening in chest }\end{array}$ \\
\hline Nimesulide & 1 & Burning micturition,throat pain and Steven Johnson syndrome. \\
\hline Diclofenac Sodium & 1 & Maculopapular rashes \\
\hline \multicolumn{3}{|c|}{ Antidepressant and antipsychotic drugs } \\
\hline Mirtazapine & 3 & Excessive sedation, vertigo and confusion \\
\hline Desvenlafaxine & 2 & Headache, anxiety and restlessness \\
\hline Olanzapine & 4 & Drowsiness and weight gain \\
\hline Quetiapine & 1 & Anxiety, Restlessness \\
\hline Etizolam & 1 & Severe Headache \\
\hline Lorazepam & 1 & Nusea, Vomiting and Dizziness \\
\hline \multicolumn{3}{|l|}{ Anticonvulsant drugs } \\
\hline Divaproex Sodium & 3 & $\begin{array}{l}\text { Gastric intolerance, vomiting, abdominal fullness, giddiness and } \\
\text { restlessness }\end{array}$ \\
\hline Lamotrigine & 1 & Steven Johnson syndrome and cutaneous ulceration \\
\hline Pregabaline & 1 & Dizziness, restlessness \\
\hline Topiramate & 1 & Generalized itching \\
\hline Phenytoin Sodium & 6 & Erythromatous maculopapular rashes \\
\hline \multicolumn{3}{|l|}{ Miscellaneous drugs } \\
\hline Iron Sucrose & 2 & Angioedema \\
\hline Multivitamins & 1 & Rashes, abdominal pain \\
\hline Tetanus Vaccine & 1 & Tonic convulsion \\
\hline
\end{tabular}


This study also shows significant level of ADR by diclofenac sodium (8\%) similar to Shrivastava MP et al. ${ }^{14}$

Table 6: Causality of reported ADR (total 174).

\begin{tabular}{|lll|}
\hline UMC-Scale & $\begin{array}{l}\text { No. of } \\
\text { reports }\end{array}$ & $\begin{array}{l}\text { \% of } \\
\text { reports }\end{array}$ \\
\hline Certain & 0 & 0 \\
\hline Probable/likely & 165 & 94.8 \\
\hline Possible & 9 & 5.2 \\
\hline Unlikely & 0 & 0 \\
\hline Conditional/Unclassified & 0 & 0 \\
\hline Unassessable/Unclassifiable & 0 & 0 \\
\hline
\end{tabular}

In our study, maximum ADRS reported were probable (94.8\%) followed by possible (5.2\%) No other ADR reported in other category of WHO-UMC System. Definite is least due to rechallenge is not possible. In contrast Sriram et al study showed that $42 \%$ were possible and $23 \%$ were probable. ${ }^{12}$

\section{CONCLUSION}

In conclusion, the skin was most affected system followed by gastrointestinal, central nervous and respiratory system. Rifampicin has caused maximum ADRs followed by zidovudine, nevirapine and diclofenac sodium. The causality analyses showed that majority of ADRs were probable $(94.8 \%)$ while remaining falls in possible $(5.2 \%)$ category.

Funding: No funding sources

Conflict of interest: None declared

Ethical approval: The study was approved by the Institutional Ethics Committee

\section{REFERENCES}

1. World Health Organization: International drug monitoring; the role of hospital. In technical report series no. 425, Geneva, Switzerland: World Health Organization; 1966:1-25.

2. Brewer T, Colditz GA, Post marketing surveillance and adverse drug reactions: current perspectives and future needs. JAMA. 1999;281(9):824-9.

3. Lobo MG, Pinheiro SM, Castro JG, Momenté VG, Pranchevicius MC. Adverse drug reaction monitoring: support for pharmacovigilance at a tertiary care hospital in northern Brazil. BMC Pharmacol Toxicol. 2013;14:5.

4. Adithan $\mathrm{C}$, The national pharmacovigilance program. Indian J Pharmacol. 2005;37:347.

5. Gor AP, Desai SV. Adverse drug reactions in the inpatients of Medicine department of a rural tertiary care teaching hospital and influence of Pharmacovigilance in reporting adverse drug reaction. Indian J Pharmacol. 2008;40(1):37-40.

6. Patidar D, Rajput MS, Nirmal NP, Savitri W. Implementation and evaluation of adverse drug reaction monitoring system in a tertiary care teaching hospital in Mumbai, India. Interdiscip Toxicol. 2013;6(1):41-6.

7. Brennan TA, Leape LL, Laird NM. Incidence of adverse events and negligence in hospitalized patients. Results of the Harvard Medical Practice Study I. N Engl J Med. 1991;324(6):370-6.

8. Murphy BM, Frigo LC. Development, implementation, and results of a successful multidisciplinary adverse drug reaction reporting program in a university teaching hospital. Hosp Pharm. 1993;28(12):1199-204.

9. Coelo HL, Arrais PSD, Parente AP, Brizeno MOB. Federal University of Ceaara, Fortaleza, Brazil. Pharmacoepidemiol Drug Safety. 2002;11(2):231-94.

10. Rajesh R, Ramesh M, Parthasarathi G. A study on adverse drug reactions related hospital admission and their management. Indian $\mathrm{J}$ Hospital Pharm. 2008;45:143-8.

11. Suh DC, Woodall BC, Shin SK, Shin SK, Hermes-De Santis ER. Clinical and economic impact of adverse drug reactions in hospitalized patients. Ann Pharmacother. 2000;34:1373-8.

12. Sriram S, Ghasemi A, Ramasamy R. Prevalence of adverse drug reactions at a private tertiary care hospital in south India. J Res Med Sci. 2011;16(1):16-25.

13. Fredy IC, Chandrashekhar S, Srinivasan R. Retrospective analysis of reported adverse drug reactions Indo Am. J. Pharm. Sci. 2015;3(1).

14. Uchit GP, Shrivastava MP, Badar VA, Navale SB, Mayabhate MM. Adverse drug reaction to antimicrobial agents in tertiary care hospital in Nagpur. J Indian Med Assoc. 2012;110(4):224-7.

15. Tandon VR, Sharma S, Khajuria V, Mahajan V, Gillani Z. Adverse drug reactions profile of antimicrobials: A 3-year experience, from a tertiary care teaching hospital of India. Indian $\mathbf{J}$ Med Microbiol. 2015;33(3):393-400.

16. Leone R, Sottosanti L, Luisa Iorio M. Drug-related deaths: An analysis of the Italian spontaneous reporting database. Drug Saf. 2008;31:703-13.

Cite this article as: Saxena $\mathrm{K}$, Tailor $\mathrm{C}$, Mehta $\mathrm{C}$, Gajera P, Srivastava SK. A retrospective analysis of adverse drug reaction reported in a tertiary care hospital. Int J Basic Clin Pharmacol 2017;6:1146-50. 